\title{
Atomic Physics in ITER - The Foundation for the Next Step to Fusion Power
}

\author{
D. P. Stotler*, R. E. Bell ${ }^{*}$, K. W. Hill ${ }^{*}$, D. W. Johnson* and F. M. Levinton ${ }^{\dagger}$ \\ *Princeton Plasma Physics Laboratory, Princeton University, P. O. Box 451, Princeton, NJ \\ 08543-0451, USA \\ ${ }^{\dagger}$ Nova Photonics, Inc., Princeton, NJ 08540, USA
}

\begin{abstract}
ITER represents the next step towards practical magnetic confinement fusion power. Its primary physics objective is to study plasmas in which the fusion power exceeds the external heating power by a factor of 5 to 10 ; its technological objectives include the use of superconducting magnets and remote maintenance. We will describe the ITER experiment and then detail the fundamental roles that will be played by atomic physics processes in facilitating the achievement of ITER's objectives. First, atoms and molecules generated by the interaction of the ITER plasma with surrounding material surfaces will impact and, in some respects, dominate the particle, momentum, and energy balances in both the adjacent and confined, core plasmas. Second, impurity radiation in the edge plasma, either from intrinsic or extrinsic species, will ensure that heat coming out from the core is spread more uniformly over the surrounding material surfaces than it would otherwise. Third, many of the diagnostics used to monitor the dense $\left(n_{e} \sim 10^{20} \mathrm{~m}^{-3}\right)$, hot $\left(\sim 1 \times 10^{8} \mathrm{~K}\right)$ core plasma leverage off of atomic physics effects.
\end{abstract}

Keywords: ITER,tokamak divertor,tokamak diagnostics

PACS: 52.55.Fa, 52.25.Ya, 52.55.Rk, 52.70.-m

\section{INTRODUCTION}

Fusion energy is envisioned to contribute significantly to the world's energy needs by the end of this century. The associated reduction in carbon dioxide emission relative to fossil fuel power plants will be important in mitigating global warming. The proposed "fast track" $[1,2]$ to fusion energy would put power on the grid in about 50 years, just when new technologies will be required to replace fossil fuel based energy [3]. Approximately the last half of this development period will be devoted to the operation of one or more demonstration power plants. The objective of these devices would be to show that fusion reactors can not only generate net electrical power, but do so economically with high levels of reliability and low activity waste products.

An aggressive, multi-component research plan will be required to establish the technical, physics, and safety basis for a demonstration power plant. One component of this plan is a facility for testing candidate fusion reactor structural materials for their ability to withstand bombardment by fusion neutrons and operate at high temperatures without experiencing a significant degradation of materials properties. This is International $\mathrm{Fu}-$ sion Materials Irradiation Facility (IFMIF) [2]. But, the more important component is a device that will demonstrate the scientific and technological feasibility of fusion power; this is the ITER experiment.

In this paper, we describe ITER in more detail and then begin to delve into the roles 
that atomic physics will play in it. Most of these processes are associated with plasmamaterial interactions. To clarify the nomenclature associated with these processes, we first briefly characterize the plasma boundary in which these interactions occur. Atomic physics processes will also play crucial roles in some of the diagnostics used to probe the core of the ITER plasma. We give examples of three such diagnostics and highlight some of the issues that must be addressed prior to their deployment on ITER.

\section{THE ITER EXPERIMENT}

ITER's primary scientific objective is to produce and control a burning fusion plasma for extended periods of time [4]. More specifically, the goal will be to achieve conditions in which the plasma heating due to the fast alpha particles resulting from deuteriumtritium fusion reactions exceeds the externally supplied heating and to maintain those conditions for a period long compared to characteristic plasma time scales. ITER will also test reactor relevant technologies such as superconducting magnets, high heat and neutron flux components, and remote maintenance.

ITER will not generate net electrical power. However, it will test power reactor blanket modules. These will be connected to steam turbines and other machinery associated with the generation of electricity, at about the $1 \mathrm{MW}$ level. The blankets will also be used to test concepts for breeding tritium; a fusion reactor will need to breed all of its tritium. Although this is not the case for ITER, it will have to recycle its tritium since each atom will pass through the plasma many times before being consumed in a fusion reaction.

The particular magnetic configuration chosen for ITER is the tokamak [2]. This choice was driven by the vast, successful experience with tokamak experiments around the world over the last forty years. The relative simplicity of the tokamak design was also a crucial consideration. However, the tokamak concept does have shortcomings that could result in fusion power plants being based on an alternative configuration [2].

The tokamak is a nearly axisymmetric torus. The principal non-symmetric aspect is the set of discrete magnetic field coils which generate the dominant toroidal magnetic field. However, with enough of these coils (order ten), the magnetic field seen by the plasma is effectively axisymmetric. With just this field, plasma ions and electrons would experience a net drift due to the radial gradient in the field and quickly find their way to the surrounding vacuum vessel. The tokamak concept resolves this problem with a secondary magnetic field in the poloidal direction generated by a toroidally directed plasma current driven by an external transformer [2]. The net result is a helically shaped magnetic field. The basic parameters describing a tokamak are its major and minor radii $R$ and $a$, respectively, its toroidal magnetic field $B_{T}$ (typically a few tesla), and its toroidal plasma current $I_{p}$ (on the order of mega-amperes). While early tokamaks utilized circular poloidal cross sections, plasmas with a more D-shaped cross section have yielded better confinement and stability properties. Additional parameters characterize the elongation and triangularity of this shape.

The helically shaped field lines trace out closed magnetic surfaces, encompassing a particular amount of toroidal or poloidal flux. The plasma electron and ion orbits are then bound to these nested, magnetic flux surfaces. Their rapid, sound speed motion along the field lines results in plasma parameters, e.g., density and temperature, which 
are to lowest order a constant on the surfaces, rendering the system essentially 1D. Plasma turbulence due to small scale instabilities driven by the plasma pressure gradient across these surfaces, as well as collisions, causes a slow, diffusive transport of particles, momentum, and energy towards the material boundaries surrounding the plasma. A significant fraction of magnetic fusion research is targeted at understanding and controlling these instabilities and the resulting transport.

The amount of fusion power produced in tokamak experiments has increased by about 10 orders of magnitude over the last forty years, culminating in experiments on the TFTR and JET devices yielding 10 and $16 \mathrm{MW}$ of fusion power, respectively, for a period of about 1 second [2]. The external heating supplied maintain the core plasma ion temperatures at the $10+\mathrm{keV}$ levels necessary for fusion was slightly more than this, so that these experiments are considered to have a "fusion gain" $\left(Q=P_{\text {fus }} / P_{\text {in }}\right)$ of slightly less than 1 . In contrast, the ITER experiment's baseline mode of operation targets $Q=10$ with $P_{\text {fus }}=500 \mathrm{MW}$ and a pulse length of about 400 s [4]. An extended mode of operation at $Q=5\left(P_{\text {fus }}=300 \mathrm{MW}\right)$ with a pulse length of $1000 \mathrm{~s}$ is also planned.

ITER's principal parameters are largely determined by these objectives and a handful of relations derived from engineering requirements and the tokamak database [5, 4]. A simple calculation, described in $[5,4]$, utilizing these relations closely estimates the actual ITER parameters that are the result of vastly more detailed engineering studies. Those parameters are [4] $R=6.2 \mathrm{~m}, a=2.0 \mathrm{~m}, I_{p}=15 \mathrm{MA}$, and $B_{T}=5.3 \mathrm{~T}$. The total plasma volume is about $850 \mathrm{~m}^{3}$; the energy in the toroidal field magnets at full strength is about $41 \mathrm{GJ}$.

The crucial decision of where to build ITER was made in June 2005 when the ITER parties agreed that the experiment should be sited in Cadarache, France. Since then, the ITER Joint Work Site in Cadarache has been opened and a public discussion of local issues has taken place. On May 24, 2006, representatives of the ITER parties, now consisting of Euratom, Japan, the United States of America, the Russian Federation, the People's Republic of China, the Republic of Korea, and India, initialed the international agreement for the construction, operation, and decommissioning of ITER. Signing by the respective governments is planned for November 21. With the addition of India, the ITER parties have committed to contributing $110 \%$ of the expected project cost, resulting in a significant contingency fund. Most of these contributions will consist of the actual ITER hardware; an agreement specifying each party's responsibilities has also been developed.

\section{THE BOUNDARY OF TOKAMAK PLASMAS}

The radial plasma particle and energy flows out of the tokamak plasma find their way to the surrounding material boundaries. To keep the core in steady state, these losses must be balanced by corresponding sources. In non-burning (pure hydrogen or deuterium) plasmas, the required power is supplied externally by the ohmic heating associated with the toroidal plasma current, energetic beams of neutral deuterium atoms, or radio frequency waves, so that $P_{\text {out }}=P_{\text {in }}$. In a plasma containing a mix of both deuterium and tritium, the fast alpha particles resulting from fusion reactions deposit $P_{\alpha}=P_{\text {fus }} / 5$ 

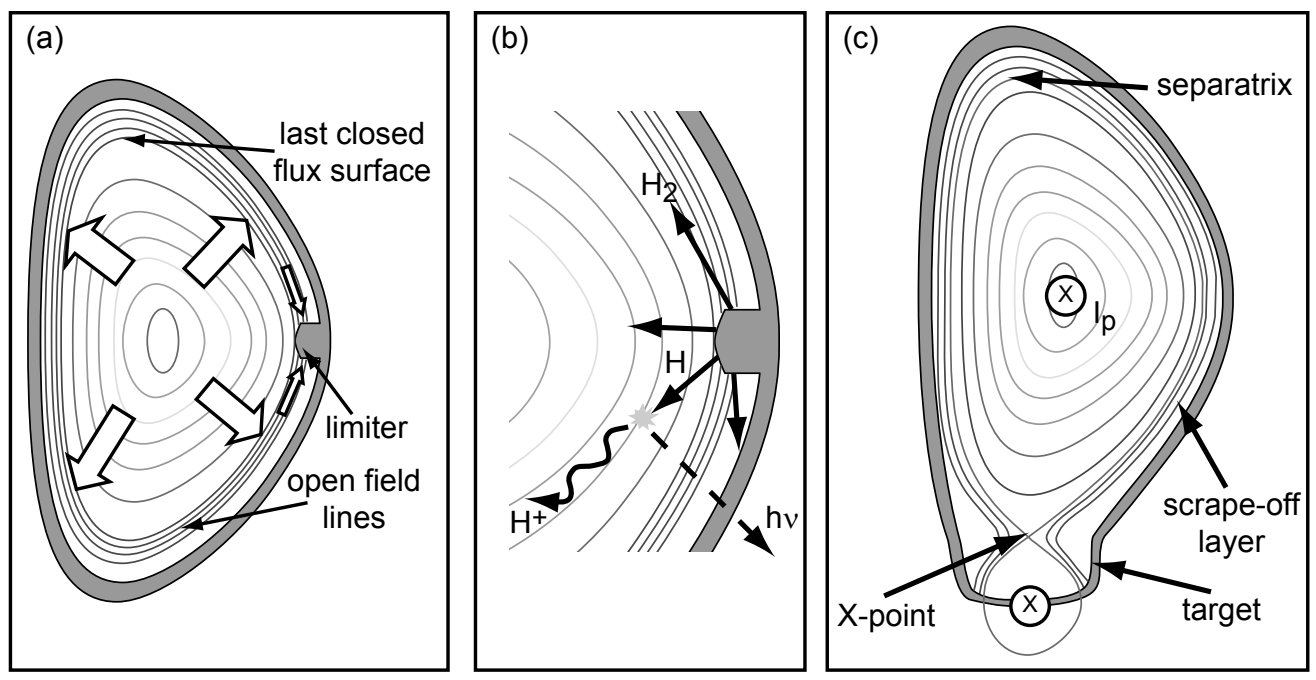

FIGURE 1. (a) Generic configuration for a tokamak limiter. (b) Schematic representation of hydrogen atoms and molecules being recycled from the limiter surface. (c) Generic configuration for a (lower single null) tokamak divertor.

directly into the plasma, giving $P_{\text {out }}=P_{\text {in }}+P_{\alpha}$. Of course, the other $80 \%$ of the fusion power carried by the $14 \mathrm{MeV}$ neutrons is captured in surrounding blankets. External particle sources such as the atoms supplied by the heating beam or by the injection of cryogenic hydrogen pellets (with inward diffusion of plasma also playing a role) can be used to replace core particle losses. But, the surrounding materials provide another very important, and complicating, source [6].

The material boundary of magnetic fusion devices is configured so that these plasma particle and energy flows interact with material surfaces at a specific location or locations, facilitating the control of the interactions. The simplest configuration is the limiter. A variety of different limiter types are possible [7], but they are functionally equivalent to the one depicted in Fig. 1(a). Namely, the limiter defines a "last closed flux surface" (LCFS), with flux surfaces beyond it consisting of open field lines which terminate at both ends on the limiter. This region outside the LCFS is referred to as the "scrape-off layer". Once the plasma particles and energy diffuse across the LCFS, they flow rapidly along those field lines and strike the limiter.

Because the plasma electrons and ions recombine at the limiter surface and their energy and momenta are absorbed by it, the limiter acts as a sink for plasma particles, momentum, and energy. It does not, however, act as a sink for mass [7]. That is, some fraction of the resulting atoms recoil off of limiter substrate atoms and head back into the plasma. Others are absorbed, but eventually bond with a second atom, forming a hydrogen molecule that is chemically unbound and able to freely leave the surface [Fig. 1(b)]. In steady state, the flux of these atoms and molecules must balance the incoming plasma flows. Because they are neutral, the atoms and molecules travel freely across field lines and can be ionized near or far from the limiter. Through this recycling process, the plasma can refuel itself. Prior to being ionized, recycled atoms can be excited and then decay radiatively. These photons (e.g., the Balmer- $\alpha$ visible transition) 
provide a straightforward means of diagnosing plasma recycling.

Physical and chemical "sputtering" of atoms and molecules off of the limiter substrate $[7,8]$ is particularly deleterious in this configuration since those neutrals have ready access to the closed flux surfaces and, via diffusion, to the core plasma. For this reason, an alternative configuration known as the divertor [Fig. 1(c)] was developed [7]. One or more coils are added at the bottom (and / or top) of the vacuum vessel By driving a current in these coils in the same direction as the plasma current, a null is created in the poloidal magnetic field. The magnetic flux surface which passes through this null is called the separatrix and the null point the X-point. In this configuration, the separatrix becomes the LCFS. The portion of the vacuum vessel below the $\mathrm{X}$-point is referred to as the divertor. As in the limiter configuration, the open field lines in the scrape-off layer eventually strike a material surface, called the divertor target. The key virtue of the divertor is that this point of plasma-material interaction is physically removed from the closed flux surfaces. Moreover, the neutral gas created by the recycling process is effectively concentrated in the divertor, facilitating pumping. The primary drawback is that the plasma heat fluxes are also concentrated.

ITER's magnetic configuration is a lower single null conceptually similar to that shown in Fig. 1(c). One distinction between ITER and most devices operated heretofore is its use of different materials for its plasma facing surfaces. The main chamber walls will be made of beryllium. The divertor target strike plates will be made of carbon fiber composite; the rest of the divertor will be tungsten $[4,5]$.

Present day devices can achieve divertor plasma parameters comparable to or even exceeding (in the case of the high density Alcator C-Mod discharges, e.g., [6]) those expected for ITER. Typical parameter ranges explored in those experiments are $n_{e}=$ $10^{20}-10^{21} \mathrm{~m}^{-3}, T_{e}=0.1-100 \mathrm{eV}$, atom density $n_{\mathrm{H}}=10^{19}-10^{20} \mathrm{~m}^{-3}$, and neutral gas pressures (in gas plenum) $P_{D_{2}}=0.1-10 \mathrm{~Pa}$. Of course, the parameters in a given tokamak discharge vary in space, with the highest $n_{e}$ and lowest $T_{e}$ usually found in the private flux region between the divertor strike points and just below the X-point.

\section{ATOMIC PHYSICS IN THE EDGE AND DIVERTOR PLASMAS}

\section{Recycling}

Estimates of ITER divertor parameters are the result of sophisticated codes that simulate the transport of plasma and neutral species in the tokamak edge and divertor regions. The characterization of turbulent plasma transport across magnetic flux surfaces in these codes is largely empirical; the models used to describe atomic physics processes are much more well founded in comparison. These processes can be modeled in detail by kinetic neutral transport codes such as EIRENE [6] and DEGAS 2 [9] due to the flexibility provided by the Monte Carlo algorithm that they employ. In these simulations, and in reality, atomic physics processes mediate the exchange of mass, momentum, and energy between the plasma and neutral species.

Electron impact ionization and recombination of $\mathrm{H}$ and $\mathrm{H}^{+}$(" $\mathrm{H}$ " refers to any of the three hydrogen isotopes) is handled by a collisional radiative model since tokamak divertor parameters typically fall between coronal and local thermodynamic equilibrium. 
One distinction from other applications, such as in astrophysics, is that ionization and recombination are not in equilibrium [10]. Rather, the tokamak plasma is usually dominated by ionization because of the recycling process. Elastic scattering of $\mathrm{H}$ and $\mathrm{H}_{2}$ on $\mathrm{H}^{+}$can be simulated with the fully quantal differential scattering cross sections computed by Krstic [11,9]. The most important effect of this scattering is to increase $\mathrm{H}_{2}$ energies (relative to their initial, wall temperature energies) and, hence, their mean free path length.

The basic set of $\mathrm{H}_{2}$ and $\mathrm{H}_{2}^{+}$dissociation and ionization processes given in [12] sufficed for the lower density limiter and divertor scrape-off layer plasmas of twenty years ago. However, the higher $n_{e}$ and lower $T_{e}$ values attained in some present day devices, and expected for ITER, result in longer lifetimes for molecular species and, thus, additional processes. In particular, vibrational excitation becomes significant under these conditions, so that ion conversion $\left[\mathrm{H}_{2}(v)+\mathrm{H}^{+} \rightarrow \mathrm{H}_{2}^{+}+\mathrm{H}\right]$ and species such as $\mathrm{H}_{3}^{+}$ and $\mathrm{H}^{-}$must be considered. The work described by Fantz et al. [13] indicates the need for incorporating these processes into a comprehensive $\mathrm{CR}$ model for $\mathrm{H}, \mathrm{H}^{+}, \mathrm{H}_{2}$, and $\mathrm{H}_{2}^{+}$. Janev et al. have recently reviewed the current state of the data required for such a model [14]. They conclude that although much of these data are available, additional data are still needed [14].

Neutral-neutral elastic scattering of $\mathrm{H}$ and $\mathrm{H}_{2}$ (i.e., gas viscosity in fluid terminology) is also significant under these high density conditions [15], as is trapping of Lyman- $\alpha$ radiation by $\mathrm{H}$ atoms. An approximate technique for handling the nonlinearity introduced by the former appears to work well [15]. However, simulating the latter requires selfconsistently treating the interactions between the plasma, gas, and radiation fields [16]. The problem is complicated even further by the various phenomena that determine the shape of the emission lines (Doppler and Stark broadening, Zeeman splitting, etc.) [17]. The paper by Reiter presented at the previous ICAMDATA conference [6] describes an example simulation of an Alcator C-Mod discharge in which all of the above processes play a role.

The chemical sputtering process mentioned previously leads to another complex set of species and reactions. Namely, the hydrocarbons eroded by chemical sputtering off of graphite surfaces can be redeposited in various locations in the tokamak vacuum vessel. As this occurs, hydrogen atoms can be effectively trapped, making their removal difficult. Deuterium-tritium experiments on the TFTR and JET tokamaks yielded carefully analyzed data on the rate at which this trapping, of tritium in particular, occurs [8]. Extrapolation of those data to ITER conditions suggest that the resulting accumulation of tritium in the vessel could exceed specified safety limits [8] in $\sim 100$ discharges! Unfortunately, the mechanisms by which these hydrocarbons are created, transported, and deposited are not completely understood, although these are topics that are being aggressively studied (as are techniques for removing the deposited films or purging the tritium from them). Making progress on this front will hinge on having a firm understanding of the atomic physics of these species. Recent work by Janev et al. has led the way in this area with extensive monographs covering $\mathrm{CH}_{y}, \mathrm{C}_{2} \mathrm{H}_{y}$, and $\mathrm{C}_{3} \mathrm{H}_{y}[18,19]$; these data have also been incorporated into 0-D reaction analysis tool [20]. As in the case of vibrationally excited molecules, the real need from the perspective of a modeler is for a simple, comprehensive model that can be readily incorporated into existing simulation codes. 


\section{Radiating Mantle}

Atomic physics processes will play another significant role in ITER: spreading the power flowing out of the core over a larger fraction of the first wall. If ITER is operating at $Q=10$ with $P_{\text {fus }}=500 \mathrm{MW}, P_{\alpha}+P_{\text {in }}=150 \mathrm{MW}$ will, in steady state, be flowing from the core plasma and exhausted to the surrounding materials. In the absence of any other effects, this power will diffuse across the separatrix and then travel rapidly along the field lines to the divertor targets. The actual area on those targets absorbing that power is governed by the width of the scrape-off layer, which in turn is determined by the plasma through the competition between the fast parallel flow and the radial (turbulent) transport. As in the case of core plasma transport, the latter is not well understood, and we can only estimate this width in ITER. An extrapolation of empirical models leads to values as small as $2 \mathrm{~m}^{2}$ [21]. Hence, the divertor target plates would be subject to a steady heat flux of $75 \mathrm{MW} / \mathrm{m}^{2}$, comparable to the radiant heat flux at the surface of the Sun!

Existing materials, with active cooling, are only able to sustain steady heat fluxes in the range of $10-20 \mathrm{MW} / \mathrm{m}^{2}$, however [5]. Larger heat fluxes could result in damage (e.g., melting) to the material or intolerable influxes of impurities into the plasma. The expectation is that in ITER radiation from impurities will disperse $\sim 75 \%$ of the heat flow over a large fraction of the first wall, reducing the heat load on the divertor targets to a tolerable level. If intrinsic impurities (e.g., carbon) are unable to do this effectively, extrinsic impurities will be injected into the ITER plasma.

The location at which these impurities radiate must be carefully considered. If the impurities radiate too much in the core, they will do so at the expense of the fusion reactions [5]. If the radiation is too close to the divertor targets, the heat load will not be adequately reduced. An ideal arrangement is for a radiating "mantle" just inside the last closed flux surface. Even there, the amount of power that can be radiated without a deleterious impact on core plasma confinement is limited [5]. More detailed modeling predicts that radiation from inside the separatrix, and in the scrape-off layer and divertor will reduce divertor target heat loads to less than $10 \mathrm{MW} / \mathrm{m}^{2}$ [22]. To prevent buildup of extrinsic impurities on plasma facing materials, "recycling" elements such as noble gases (neon, argon, krypton, xenon) or nitrogen are being considered. Since these are gases, they are also easier to introduce into the vacuum vessel. Although the atomic physics models used in these simulations undoubtedly have many uncertainties, they are likely to be insignificant compared with those associated with extrapolating the empirical transport models to ITER.

\section{ATOMIC PHYSICS IN CORE DIAGNOSTICS}

Another important role for atomic physics in ITER is in diagnosing the core plasma conditions. For example, some means of tracking impurity species, such as helium ash or seeded impurities, will be needed. The only means of generating photons in the hot core plasma, $T_{e}>10 \mathrm{keV}$, is to either rely on high $Z$ atoms (intrinsic or extrinsic) or inject low $Z$ atoms at high enough energy that they are able to penetrate to the core. We will briefly describe diagnostics of both types. 


\section{Charge Exchange Recombination Spectroscopy}

Charge exchange recombination spectroscopy (CXRS) will give data on impurity ion densities (via emission intensity), temperature (via Doppler broadening), and flow velocities (via Doppler shift) [23, 24]. The technique relies on an energetic neutral hydrogen (again, referring to no particular isotope) beam which undergoes charge exchange with fully stripped impurity ions in the core plasma, $\mathrm{H}+\mathrm{A}^{+q} \rightarrow \mathrm{H}^{+}+\left[A^{+(q-1)}\right]^{*}$. The specification of fully stripped ions follows, first, because that is what one expects to find in the core plasma and, second, because it renders the resulting product impurity ion hydrogen-like, simplifying its atomic physics. Visible lines are used so that the emission spectrum can be easily measured. While many CXRS systems are based on the tokamak's heating neutral beam, a dedicated diagnostic beam provides greater control and, by virtue of its smaller cross section, better spatial resolution. CXRS systems on existing tokamaks mostly utilize carbon as the impurity species.

Interpretation of the resulting spectra would ideally be done using cross sections for charge exchange into individual $n$ and $l$ states of the $\mathrm{A}^{+(q-1)}$ atom. Cross sections at this level of detail are usually calculated theoretically; although most data being used with existing CXRS systems have been validated to some degree by experimental measurements. The analysis also needs to account for mixing of nearly degenerate states by the plasma environment [23]. Rates for the cascade of the excited states towards the ground state and the corresponding emission are likewise required.

Additional effects can complicate interpretation of the diagnostic signal. Contributions from "halo" atoms [23] and "plume" ions [24] must be factored in. The sources of $\mathrm{H}^{+}$used in the neutral beam injectors create $\mathrm{H}_{2}^{+}$and $\mathrm{H}_{3}^{+}$ions as well, resulting in significant fractions of beam atoms at $1 / 2$ and $1 / 3$ of the full beam energy. The beam atoms can also be in excited states [23]. Even though the fractions may be small, the corresponding charge exchange cross sections are much larger, so that these their contributions cannot be ignored. More subtle effects such as the gyro-orbit dependence noted by Bell [25] also arise.

The CXRS system contemplated for ITER is part of a comprehensive package of active beam spectroscopy diagnostics [26,27]. One potential improvement to this system would be the use of multiple views to permit the elimination of gyro-orbit effects [25]. Additional $(n, l)$ resolved cross sections are needed, particularly at the lower energies typical of the $1 / 2$ and $1 / 3$ beam energy components (ITER's heating beam has an energy of $500 \mathrm{keV} / \mathrm{amu}$; the diagnostic beam $100 \mathrm{keV} / \mathrm{amu}$ ). The greatest potential problem for ITER's CXRS system is the degradation of the first mirrors by $\mathrm{C}$ and Be coatings. Methods of calibrating and / or cleaning the mirrors in situ will have to be developed.

\section{Motional Stark Effect}

The strength of a tokamak's poloidal magnetic field cannot be computed a priori since the spatial distribution of the current is determined self-consistently by the plasma. Yet, this information is essential for the reconstruction of the magnetic equilibrium and in the testing of theories of plasma equilibrium, stability, and transport. The "Motional Stark 
Effect" (MSE) diagnostic is intended to provide this information via a spatially resolved measurement of the magnetic field's orientation. Like CXRS, MSE relies on a heating or diagnostic beam. In contrast, however, the latter utilizes the Balmer- $\alpha$ line resulting from plasma excitation of the beam atoms. In the frame of the beam, the atoms experience an electric field $\mathbf{E}=\mathbf{v}_{\text {beam }} \times \mathbf{B}$. The associated Stark effect yields a wavelength splitting of several angstroms and polarization of the emitted radiation that can be exploited to yield the desired information [28]. As with CXRS, a single beam with multiple sight lines provides a full radial profile of the field line pitch.

The effect of coatings on the ITER diagnostic mirrors will be even more problematic for the MSE system than for CXRS since the coatings may prevent accurate polarimetry altogether. Since they will affect line shifts much less than the polarimetry, an approach based on precision spectrometry is being considered. A correspondingly detailed [i.e., $(n, l)$ resolved] collisional radiative model will be required. A second alternative based on the measurement of the ratio of $\pi$ and $\sigma$ lines has been proposed [27, 26], although it will require isotropic reflection throughout the optical system.

\section{X-Ray Crystal Spectroscopy}

Independent measurements of plasma quantities are useful precisely because of difficulties like those noted in the previous sub-sections. X-ray crystal spectroscopy is planned for use on ITER to give another measurement of impurity ion temperatures and flow velocities complementary to that provided by CXRS. In contrast to CXRS, this system will study emission lines from high $Z$ impurities such as $\mathrm{Kr}$, Ar, or W. In particular Barnsley et al. [29] discuss the use of H- and He-like $\mathrm{Kr}$, $\mathrm{Ar}$, and Fe in ITER. The data required to interpret the expected spectra for these atoms are available. The amount of impurity that can be injected into the plasma and, hence the brightness of the measured emission, is limited by that species' contribution to the total radiated power [29]. Another consideration is the relative amount of noise due to free-free and freebound continuum emission [29]. Tungsten is an intrinsic impurity since it is used in the divertor target plates. Better data on W energy levels and emission rates are needed. Experiments involving W on the ASDEX-Upgrade (underway now) and JET (near future) will allow models for W spectroscopy to be tested well in advance of ITER operation.

\section{SUMMARY}

That ITER is an ambitious experience is readily acknowledged. In the process of designing and preparing to build it, the fusion community is testing existing models and developing new ones more aggressively than ever before. This is especially true for the edge and divertor plasmas. It is here that our knowledge of atomic physics is crucial to the project's success, whether in dealing with plasma-wall interactions or in taming problems like tritium retention or excessive heat loads. Atomic physics is also essential for several of ITER's core diagnostics; we will need to be confident in interpreting their signals to gauge ITER's performance. 


\section{ACKNOWLEDGMENTS}

The author would like to acknowledge useful discussions with D. Reiter (IPP-Jülich) and R. Schneider (IPP-Greifswald). This work has been supported by U.S. DOE Contract DE-AC02-76CHO3073.

\section{REFERENCES}

1. C. L. Smith, The fast track to fusion power (2004), presented at the 20th IAEA Fusion Energy Conference, Vilamoura, Portugal, November 1 - 6, 2004.

2. International Fusion Research Council, Nucl. Fusion 45, A1-A28 (2005).

3. R. H. Socolow, and S. W. Pacala, Sci. Am. 295, 50-57 (2006).

4. I. T. B. Editors, Iter technical basis, Tech. Rep. ITER EDA Documentation Series No. 24, IAEA, Vienna (2002); see also http: / / www. iter. org.

5. ITER Physics Basis Editors, Nucl. Fusion 39, 2137-2638 (1999).

6. D. Reiter, et al., "The role of atomic and molecular processes in magnetic fusion plasmas," in Atomic and Molecular Data and Their Applications, AIP Conf. Proc. 771, American Institute of Physics, 2005, New York.

7. P. C. Stangeby, The Plasma Boundary of Magnetic Fusion Devices, Institute of Physics Publishing, Philadelphia, 2000.

8. G. Federici et al., Nucl. Fusion 41, 1967-2137 (2001).

9. D. P. Stotler, et al., J. Nucl. Mater. 290-293, 967-971 (2001).

10. D. Reiter, "Neutral gas transport in fusion devices: Atomic and surface data aspects," in Atomic and Plasma-Material Interaction Processes in Controlled Thermonuclear Fusion, edited by R. K. Janev, and H. W. Drawin, Elsevier Science Publishers B.V., 1993, pp. 243-266.

11. P. S. Krstic, and D. R. Schultz, At. Plasma-Mater. Data Fus. 8, 1 (1998), supplement to the journal Nucl. Fus.

12. R. K. Janev, W. D. Langer, K. Evans, Jr., and D. E. Post, Jr., Elementary Processes in HydrogenHelium Plasmas, Springer Series on Atoms and Plasmas, Springer-Verlag, New York, 1987.

13. U. Fantz, D. Reiter, B. Heger, and D. Coster, J. Nucl. Mater. 290-293, 367-373 (2001).

14. R. K. Janev, D. Reiter, and U. Samm, Collision processes in low-temperature hydrogen plasmas, Tech. Rep. Jül-4105, Forschungszentrum Jülich (2003).

15. A. S. Kukushkin, et al., Nucl. Fusion 45, 608-616 (2005).

16. D. Reiter, S. Wiesen, and M. Born, Plasma Phys. Control. Fusion 44, 1723-1737 (2002).

17. M. L. Adams, and H. A. Scott, Contrib. Plasma Phys. 44, 262-267 (2004).

18. R. K. Janev, and D. Reiter, Phys. Plasmas 8, 4071 (2002).

19. R. K. Janev, and D. Reiter, Phys. Plasmas p. 780 (2004).

20. http://www.eirene.de/eigen/index.html

21. A. Herrmann, et al., J. Nucl. Mater. 313-316, 759-767 (2003).

22. A. S. Kukushkin, and H. D. Pacher, Plasma Phys. Control. Fusion 44, 931-943 (2002).

23. R. C. Isler, Plasma Phys. Control. Fusion 36, 171-208 (1994).

24. R. J. Fonck, D. S. Darrow, and K. P. Jaehnig, Phys. Rev. A 29, 3288 (1984).

25. R. E. Bell, and E. J. Synakowski, "New understanding of poloidal rotation measurements in a tokamak plasma," in Atomic Processes in Plasmas: Twelfth Topical Conference, edited by R. C. Mancini, and R. A. Phaneuf, AIP Conf. Proc. 547, American Institute of Physics, New York, 2000.

26. A. Malaquias, et al., Rev. Sci. Instrum. 75, 3393-3398 (2004).

27. M. von Hellermann, et al., Rev. Sci. Instrum. 75, 3458-3461 (2004).

28. F. M. Levinton, S. H. Batha, M. Yamada, and M. C. Zarnstorff, Phys. Fluids B 5, 2554-2561 (1993).

29. R. Barnsley, et al., Rev. Sci. Instrum. 75, 3743-3746 (2004). 